heterosexual males. Our findings imply that FPL are not useful in identifying HPV infections with a high transmission potential in this population.

Disclosure No significant relationships.

\section{P521 INCREASES IN THE ESTIMATED NUMBER OF REPORTED GONORRHEA CASES AMONG MEN WHO HAVE SEX WITH MEN (MSM): THE ROLE OF TESTING}

${ }^{1}$ Austin Williams, ${ }^{1}$ Emily Weston*, ${ }^{2}$ Thomas Gift, ${ }^{1}$ Elizabeth Torrone. ${ }^{1}$ US Centers for Disease Control and Prevention, Division of STD Prevention, Atlanta, USA; ${ }^{2}$ Centers for Disease Control and Prevention, Division of STD Prevention, Atlanta, USA

\subsection{6/sextrans-2019-sti.599}

Background Interpreting trends in rates of reported cases of gonorrhea is difficult because infections, particularly extra-genital infections, are often asymptomatic and increased screening coverage will result in increased case detection. In the absence of national data on screening coverage among men who have sex with men (MSM), we estimated trends in MSM testing in the United States given a range of positivity estimates.

Methods We estimated the number of tests that would have had to occur to identify the estimated number of reported gonorrhea cases among MSM in a given year. The number of tests was calculated by dividing the annual number of estimated cases among MSM (based on sentinel surveillance) by estimated test positivity among MSM (based on published literature). We calculated the estimated number of tests under both low positivity (3.5\%) and high positivity (10\%), as well as the effect of stable and changing positivity during 20162017.

Results The estimated number of reported gonorrhea cases among MSM increased by 17.9\% during 2016-2017 (from 163,537 to 192,740 ). We estimated that 1.9 million (assuming $10 \%$ positivity) to 5.5 million (assuming $3.5 \%$ positivity) tests performed among MSM would be needed to detect the estimated number of gonorrhea cases among MSM in 2017. This represents an increase of approximately 290,000 to 830,000 tests over the estimated number of tests conducted in 2016. Alternatively, if the number of tests was stable over time, a 0.6 to 1.8 percentage point increase in positivity from 2016 to 2017 would be consistent with the increase in observed cases.

Conclusion We provide a framework to inform trends in case rates by analyzing the impact of changes in positivity and testing over time. Our analysis implies that estimated increases in reported gonorrhea cases among MSM likely resulted from both increased screening and increased incidence.

Disclosure No significant relationships.

\section{P522 DISPARITIES IN HIVISTI TESTING AND DIAGNOSIS AMONG URBAN AND NON-URBAN US MEN WHO HAVE SEX WITH MEN FROM 2013 TO 2017}

Maria Zlotorzynska*, Travis Sanchez, Patrick Sullivan. Emory University Rollins School of Public Health, Department of Epidemiology, Atlanta, USA

\subsection{6/sextrans-2019-sti.600}

Background Most data about HIV/STI testing and diagnosis among US men who have sex with men (MSM) comes from urban areas, though the majority of the population resides outside of these areas. Since 2013, the American Men's Internet Survey (AMIS) has conducted annual nationwide online behavioral surveillance of $\geq 10,000$ US MSM.

Methods Participants age $15+$ were recruited through online advertisements. County urban/rural categories were based on National Center for Health Statistics classification. Poisson models using generalized estimating equations tested associations between urban/rural category and HIV testing, STI (syphilis, gonorrhea, chlamydia) testing and STI diagnoses in the past 12 months. All models controlled for survey year, age, race/ethnicity, insurance, HIV status (except model for HIV testing) and recruitment source.

Results From 2013 through 2017, 49,903 completed surveys were collected: $42.4 \%$ MSM from urban counties, $20.5 \%$ suburban, $28.3 \%$ small/medium metro, and $8.8 \%$ rural. STI testing was more prevalent in urban counties (50.2\%) compared to suburban $(37.8 \%, p<0.0001)$, small/medium metro $(35.6 \%, p<0.0001)$ and rural $(27.8 \%, p<0.0001)$ counties. STI diagnoses were more prevalent in urban counties (13.4\%) compared to suburban $(8.1 \%, p<0.0001)$, small/ medium metro $7.5 \%, p<0.0001)$ and rural $(5.4 \%$, $p<0.0001)$ counties. Among HIV-negative/unknown status MSM, HIV testing was more prevalent among MSM from urban counties (61.9\%) compared to suburban $(52.3 \%$, $p<0.0001)$, small/medium metro $(50.6 \%, p<0.0001)$ and rural $(43.6 \%, p<0.0001)$ counties. Significant trends over time were observed in HIV testing for all counties, while STI testing only increased in urban and small/medium metro counties. STI diagnoses increased significantly in all but rural counties.

Conclusion Urban/rural disparities in HIV/STI testing and STI diagnoses were found in a multi-year national sample of US MSM. These findings likely reflect disparate geographical distribution of healthcare access and resources. If these disparities cannot be adequately addressed in programs that reach underserved areas, nationwide HIV/STI prevention goals for MSM will not likely be met.

Disclosure No significant relationships.

\section{P523 URETHRAL MICROBIOTA IN IDIOPATHIC NON- GONOCOCCAL URETHRITIS (NGU) IN MEN WHO HAVE SEX WITH MEN AND MEN WHO HAVE SEX WITH WOMEN}

${ }^{1}$ Sujatha Srinivasan*, ${ }^{2}$ Laura Chambers, ${ }^{3}$ Ken Tapia, ${ }^{4}$ Noah Hoffman, ${ }^{1}$ Matthew Munch, ${ }^{5}$ Jennifer Morgan, ${ }^{1}$ Daniel Domogala, ${ }^{6} \mathrm{M}$ Lowens, ${ }^{4}$ Sean Proll, ${ }^{7} \mathrm{M}$ Huang, ${ }^{1}$ Keith Jerome, ${ }^{4}$ Matthew Golden, ${ }^{8}$ James Hughes, 'David Fredricks, ${ }^{2}$ Lisa Manhart. ${ }^{1}$ Fred Hutchinson Cancer Research Center, Vaccine and Infectious Disease Division, Seattle, USA; ${ }^{2}$ University of Washington, Epidemiology, Seattle, USA; ${ }^{3}$ University of Washington, Global Health, Seattle, USA; ${ }^{4}$ University of Washington, Medicine, Seattle, USA; ${ }^{5}$ Public Health - Seattle and King County, Seattle, USA; ${ }^{6}$ Public Health - Seattle and King County, HIVISTD Program, Seattle, USA; ' University of Washington, Laboratory Medicine, Seattle, USA; ${ }^{8}$ University of Washington, Biostatistics, Seattle, USA

10.1136/sextrans-2019-sti.601

Background NGU is common with no known etiology in $\sim 50 \%$ of cases. We evaluated the association of urethral bacteria with NGU among men who have sex with men (MSM) and men who have sex with women (MSW).

Methods Urine samples were collected from MSM and MSW attending a Seattle STD Clinic and enrolled in a cross-sectional study. Chlamydia trachomatis (CT) and Mycoplasma genitalium (MG) were detected by TMA (Aptima), and adenovirus, HSV-1 and HSV-2 by PCR. NGU was defined as having urethral 
symptoms or visible discharge and $\geq 5 \mathrm{PMNs}$ /high powered field (HPF). Absence of CT, MG, adenovirus, and HSV was considered as idiopathic NGU. Men without NGU had no urethral symptoms, no discharge, and $<5 \mathrm{PMNs} / \mathrm{HPF}$. Broad-range $16 \mathrm{~S}$ rRNA gene PCR with deep sequencing was used to characterize the urethral microbiota. Compositional lasso analysis of bacterial taxa was conducted to identify associations between bacteria and NGU; beta coefficients $(\beta)$ giving change in probability of NGU per $\log 2$ change in relative abundance are reported.

Results Of 434 (199 MSM, 235 MSW) urine samples, 330 yielded sequence data. NGU+ men were less likely to yield sequence data $(70 \%$ vs $84 \%$, Fisher's $\mathrm{p}=0.001)$. Of 328 men with $\geq 1000$ sequence reads/sample, 95 MSM (44 NGU+) and 143 MSW (46 NGU+) were negative for CT, MG, adenovirus, and HSV. Higher relative abundances of Haemophilus influenzae $(\beta=0.0139)$ and Mycoplasma penetrans $(\beta=0.0095)$ were positively associated with idiopathic NGU in MSM, while $H$. influenzae was positively associated with idiopathic NGU in MSW $(\beta=0.0184)$. The model also identified bacterial species that were negatively associated with NGU in MSM and MSW. Notably, Lactobacillus iners was negatively associated with idiopathic NGU in MSW ( $\beta=-0.0006)$ but not MSM.

Conclusion Different bacterial species are associated with NGU in MSM and MSW. We identified two bacterial species infrequently detected in the male urethra as positively associated with NGU. Disclosure No significant relationships.

\section{P524 GAYS, GOVERNMENT AND BIG DATA: SHOULD ROUTINE HEALTH RECORDS INCLUDE SEXUAL ORIENTATION?}

\begin{abstract}
${ }^{1}$ Peter Saxton*, ${ }^{2}$ Jeffery Adams, ${ }^{3}$ John Fenaughty, ${ }^{1}$ Daniel Exeter, ${ }^{4}$ Andrew Sporle. ${ }^{1}$ University of Auckland, School of Population Health, Auckland, New Zealand; ${ }^{2}$ Massey University, ShoreWhariki Research Centre, Auckland, New Zealand; ${ }^{3}$ University of Auckland, School of Counselling, Human Services and Social Work, Auckland, New Zealand; ${ }^{4}$ University of Auckland, Department of Statistics, Auckland, New Zealand
\end{abstract}

\subsection{6/sextrans-2019-sti.602}

Background Sexual orientation minorities continue to experience poorer outcomes in sexual health, mental health and addictions. Despite clear information needs, routine data identifying gay, lesbian and bisexual (GLB) individuals are seldom collected by governments, rendering these populations invisible. In New Zealand (NZ), everyone is assigned a unique National Health Index (NHI) number used across all health systems to improve clinical and public health decision-making. In 2017 the NZ Ministry of Health proposed adding sexual orientation and other fields to NHI, however sexual orientation was rejected after consultation. We sought to better understand these viewpoints.

Methods We used the Official Information Act (OIA) to request the complete list of submitters, their support or opposition for adding sexual orientation and written comments. We allocated submitters to six groupings: Government $(G)$; Health provider including District Health Boards (H); NGO or civil society (N); Academic (A); Data management firm (D) or Unspecified (U). Submitters did not necessarily represent the official views of their organisation. We present descriptive summaries and feedback themes.
Results 130 submissions were received. Overall 27 supported sexual orientation in NHI and 35 were opposed, the remainder being neutral or conditional. Support by grouping (high to low) was: Academic (100\%); Government (56\%); Unspecified (44\%); NGO (40\%); Health (36\%) and Data firm (25\%). Supportive reasons included: service planning; evidence-based policy; equity; GLB-specific health delivery (e.g. HPV vaccines, HIV and STI screening, pre-exposure prophylaxis); normalisation; health workforce development. Opposing reasons included: sexual orientation being irrelevant to clinical decision-making; classification challenges (e.g. sexual orientation fluidity); data quality; privacy; discrimination.

Conclusion Barriers to sexual orientation data collection include practical concerns but also well-meaning paternalism and heteronormative assumptions. Better information about NHI uses and protections, data governance, and healthcare service obligations to GLB communities, may improve support. Disclosure No significant relationships.

\section{P525 PREVALENCE OF MYCOPLASMA GENITALIUM BY ANATOMICAL SITE IN MEN WHO HAVE SEX WITH MEN: A SYSTEMATIC REVIEW AND META-ANALYSIS}

${ }^{1}$ Rosie Latimer*, ${ }^{2}$ Hannah Shilling, ${ }^{1}$ Lenka Vodstrcil, ${ }^{2}$ Dorothy Machalek, ${ }^{3}$ Christopher Fairley, ${ }^{1}$ Tim Read, ${ }^{3}$ Eric Chow, ${ }^{3}$ Catriona Bradshaw. 'Monash University, Central Clinical School, Carlton, Australia; ${ }^{2}$ The Royal Women's Hospital, Centre for Women's Infectious Disease Research, Parkville, Australia; ${ }^{3}$ Alfred Health, Melbourne Sexual Health Centre, Carlton, Australia

\subsection{6/sextrans-2019-sti.603}

Background With the current debate over testing and screening for Mycoplasma genitalium (MG) in various populations, more information on the prevalence of $\mathrm{MG}$ is needed particularly in populations at high risk of sexually transmitted infections, such as men who have sex with men (MSM). We assessed the available data on the prevalence of MG in MSM across three anatomical sites: the urethra, pharynx and rectum.

Methods Ovid Medline, PubMed, Embase were searched for all peer-reviewed studies published until 1st June 2018 (in addition to conference proceedings from 2015), that reported prevalence of MG (using nucleic acid amplification testing) in the urethra, rectum and/or pharynx in at least 50 MSM. Data were extracted by anatomical site, symptom and HIV status. Pooled estimates (95\% confidence intervals [CIs]) were calculated using random effects meta-analysis. Subgroup analyses were performed to assess heterogeneity between studies.

Results Forty-six studies met inclusion criteria. The overall prevalence of $\mathrm{MG}$ at any site was $5.8 \% \quad(4.5-7.3 \%$, $\mathrm{I} 2=95.0 \%)$. MG prevalence was 4.6\% (3.0-6.4\%, I2=94.4\%) in the urethra, $6.1 \%(4.5-7.9 \%, \mathrm{I} 2=89.0 \%)$ in the rectum, and $1.0 \%(0.0-5.1 \%, \mathrm{I} 2=96.0 \%)$ in the pharynx. Pooled estimates of MG prevalence were higher among HIV-positive compared with HIV-negative men $\mathbf{( 9 . 0 \%}$ [5.2-13.4\%, $\mathrm{I} 2=90.7 \%]$ versus $5.7 \% \quad[3.5-8.2 \%, \mathrm{I} 2=93.1 \%], \mathrm{p}=0.019)$, and among symptomatic men compared to asymptomatic men $(9.2 \% \quad[6.2-12.7 \%, \quad \mathrm{I} 2=87.3 \%]$ versus $4.0 \% \quad[2.3-6.2 \%$, $\mathrm{I} 2=90.7], \mathrm{p}=0.003)$.

Conclusion MG is commonly detected in MSM, particularly in the urethra and rectum. Prevalence was highest in HIV 